\title{
Planar, 3D, switchable UWB frequency selective surfaces
}

\author{
Rabia Yahya ${ }^{\text {a) }}$, Akira Nakamura, and Makoto Itami \\ Department of Applied Electronics, Tokyo University of Science, \\ 6-3-1 Niijuku, Katsushika-ku, Tokyo 125-8585, Japan
}

a)rabia@itamilab.te.noda.tus.ac.jp

Abstract: In this letter, we propose the designs of new frequency selective surfaces (FSS) for ultra-wideband (UWB) applications. The proposed switchable FSS is a 3D band-pass FSS constituted with a periodic array of two layers, of crossed metallic tapered dipoles, separated by a single-layer band-stop FSS and related by twin wire transmission lines. With this configuration, two propagating paths are formed. One path through the transmission lines with a corresponding band-pass response and the second path allows the propagation through the interlayer with an associated bandstop response. By inserting an on/off switch, between the crossed tapered dipoles, a switchable UWB band-pass/stop response can be achieved.

Keywords: UWB band-pass FSS, UWB band-stop FSS, active FSS, switchable FSS

Classification: Antennas and Propagation

\section{References}

[1] B. A. Munk, Frequency Selective Surfaces: Theory and Design, John Wiley \& Sons Inc, New York, 2000. DOI:10.1002/0471723770

[2] F. Capolino, Theory and Phenomena of Metamaterials, CRC Press, Taylor and Francis Group, Boca Raton, 2009. DOI:10.1201/9781420054262

[3] First Report and Order, Revision of Part 15 of the Commission's Rules Regarding Ultra-Wideband Transmission Systems, Federal Communications Commission, ET Docket 98-153, FCC 02-48, Feb. 2002.

[4] Y. Ranga, L. Matekovits, A. R. Weily, and K. P. Esselle, “A low-profile duallayer Ultra-wideband frequency selective surface reflector," Microw. Opt. Technol. Lett., vol. 55, pp. 1223-1227, June 2013. DOI:10.1002/mop.27583

[5] Bing-yuan, X. Zheng-hui, L. Wei-ming, and R. Wu, "Ultra-wideband frequency selective surface at $\mathrm{K}$ and $\mathrm{Ka}$ band," Proc. IEEE International Conference on Microwave Technology and Computational Electromagnetics (ICMTCE), Qingdao, China, pp. 55-57, Aug. 2013. DOI:10.1109/ICMTCE. 2013.6812480

[6] W. Li, T. Zhang, G. Yang, Q. Wu, and J. Hua, "Novel frequency selective surfaces with compact structure and ultra-wideband response," Proc. AsiaPacific Symposium on Electromagnetic Compatibility (APEMC), Singapore, pp. 557-560, May 2012. DOI:10.1109/APEMC.2012.6238018

[7] W. Li, G. Yang, T. Zhang, and Q. Wu, “A novel frequency selective surface with ultra-wideband polarization selective response," Proc. $12^{\text {th }}$ Conf. IEEE International Conference on Communication Technology (ICCT), Nanjing, China, pp. 1315-1318, Nov. 2010. DOI:10.1109/ICCT.2010.5689062

[8] R. M. S. Cruz, A. G. D’Assuncao, and P. H. F. da Silva, “A new FSS design 
proposal for UWB applications," Proc. International Workshop on Antenna Technology (IWAT), Lisbon, Portugal, pp. 1-4, March 2010. DOI:10.1109/ IWAT.2010.5464645

[9] S. Kamal and B. Nader, "A frequency selective surface with miniaturized elements," IEEE Trans. Antennas Propag., vol. 55, no. 5, pp. 1239-1245, May 2007. DOI:10.1109/TAP.2007.895567

[10] B. Nader, A. Mudar, and S. Mohsen, "A low-profile third-order bandpass frequency selective surface," IEEE Trans. Antennas Propag., vol. 57, no. 2, pp. 460-466, Feb. 2009. DOI:10.1109/TAP.2008.2011202

[11] B. A. Munk, Finite Antenna Arrays and FSS, John Wiley \& Sons, New York, 2003. DOI:10.1002/0471457531

\section{Introduction}

Frequency selective surfaces are periodic arrays of metallic or slots can exhibit band-stop or band-pass response when excited by incident waves. Their behavior depends not only on the frequency but also on the angle and polarization of the incident electromagnetic waves [1]. Therefore, they have multitude features, and they are widely employed as filters, polarizers, absorbers as well as in antennas engineering. Their utilizations also include various applications such as wireless communication systems, satellite communications and for radar cross-section (RCS) reduction. All this makes FSSs a rich research topic attracts the attention of academic and industrial researchers. As a result, FSSs have been treated by several books and a lot of research has been devoted to developing and analyzing these valuable structures $[1,2]$. Over the last decades, UWB technology, as well, has attracted too much attention and imposed itself as a promising technology can easily overcome multiple limits effacing the traditional technologies, such as narrow bandwidth, slow data rate, multipath fading and interference, using the license-free UWB band which is authorized by FCC in 2002 [3]. Therefore, various UWB microwave components have been proposed. In a similar concept, an attempt to design broadband FSSs has been grown to extend the applications to UWB systems whether UWB communication systems, UWB radar or to enhance the performance of UWB microwave components. The most part of the proposed broad band-stop FSSs uses the multilayer concept to achieve flat selective response over broad bandwidth $[4,5]$. Although this technique is effective to enhance the bandwidth of the FSS, it requires cascading of distanced monolayer FSSs which occupy a large space and increase the cost as well as complicating the fabrication. To overcome the size defects, broadband FSSs with periodic cells printed on a single dielectric substrate have been proposed $[6,7]$, but at the expense of the bandwidth. Furthermore, an FSS occupies one side of a substrate but with even narrower bandwidth has reported in [8]. As results, it will be convenient to introduce an UWB band-stop FSS with a single-layer. On the other hand, the proposed approaches to design band-pass FSSs are, principally, based on four techniques. The conventional method which uses resonant elements [1], the second one employs multilayer of non-resonant elements that have dimensions much smaller than the operating wavelength [9], and the third technique combines 
resonant and non-resonant elements [10] to minimize the number of the used layers. The fourth approach is receive-transmit elements connected via cables. The idea of this approach is simply that one element receives an incoming signal and transfers it via cables to the element on the other side [1]. The latter technique is superior to the others, as far as the reconfigurability is concerned. The FSS bandwidth is essentially controlled by the bandwidth of the individual elements. As a result, to design UWB FSSs based on the fourth technique, the operating bandwidth of the elements should be broadened, which can be done by using the tightly coupled concept. Tightly coupled arrays are first introduced by Munk [11] and their concept is simply used the capacitive coupling between the tightly coupled elements and the inductive effect resulting from a ground plane, placed at a distance from the array, to extend the bandwidth of the elements beyond their physical size. Consequently, a tightly coupled element above a ground plane has a wider bandwidth comparing with its bandwidth as an isolated element. Therefore, in this paper, we propose the designs of an UWB band-pass FSS based on the receive-transmit method using tightly coupled concept and a single-layer FSS with UWB band-stop response. After that, we replace the ground plane of the band-pass FSS by the single-layer FSS, which can serve as a ground plane for the tightly coupled receive-transmit elements and as an UWB reflector when the transmission lines are disconnected. In this way, a reconfigurable UWB band-pass/stop FSS is obtained. It should be mentioned that the S-parameters of the proposed FSSs were computed using CSTMWS, by considering "unit cell" boundaries, Floquet ports, and the frequency domain solver. Also, it worth to be highlighted that all the proposed FSSs are dualpolarized with similar behavior, for a normal incidence, at the both polarizations TE and TM. Therefore, only the S-parameters, for TE-polarization, are shown. The details of the proposed designs are presented in the next sections.

\section{Design of 3D band-pass FSS}

The proposed 3D FSS unit cell is constituted of dual-layer of two crossed tapered dipoles, printed on a substrate with a dielectric constant of 3.38 and a thickness of $0.508 \mathrm{~mm}$, and a ground plane placed at distance $\mathrm{h}=10 \mathrm{~mm}$ from the two substrates. The crossed tapered dipoles used as receivers are connected to the ones used as transmitters via twin transmission lines pass through slots in the center of the ground plane, as shown in Fig. 1a-b. Besides the dimensions of the crossed dipoles, the gap between the unit cells and the distance $h$ are the main parameters that control the FSS bandwidth. Also, the ground plane plays a fundamental role in increasing the bandwidth of the FSS. Without the ground plane, the small spacing between the unit cells and the connecting transmission lines are not enough to achieve UWB band-pass response. The final dimensions of the proposed UWB band-pass FSS's unit cell are $\mathrm{Wx}=\mathrm{Wy}=16.5 \mathrm{~mm}$, wx $1=2 \mathrm{~mm}$, wy $1=1 \mathrm{~mm}$, and $\mathrm{g} 1=0.3 \mathrm{~mm}$. The S-parameters of the proposed FSS are shown in Fig. 1c. From these curves, we can see clearly that a band-pass response is provided over the band from $3.1 \mathrm{GHz}$ to $10.6 \mathrm{GHz}$ with a reflection coefficient inferior to $-10 \mathrm{~dB}$. 


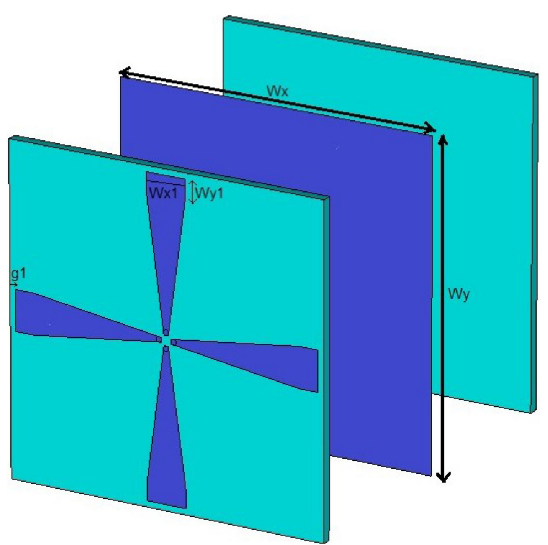

(a) Unit cell of the proposed band-pass FSS

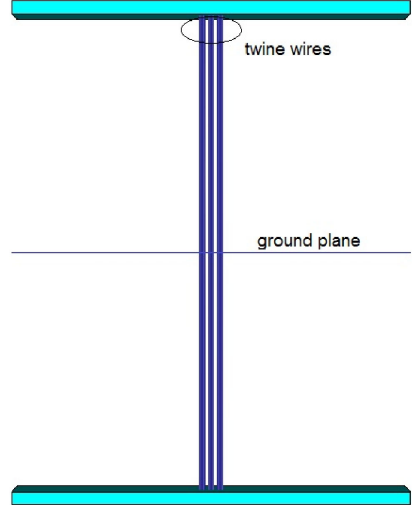

(b) Side view of the unit cell

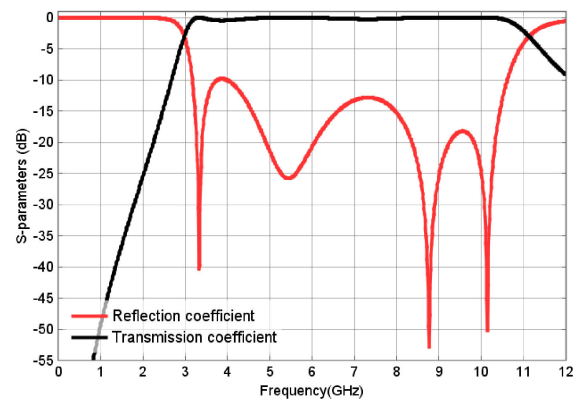

(c) S-parameters of the band-pass FSS.

Fig. 1. Structure and results of the proposed band-pass FSS.

\section{Design of the proposed single-layer band-stop FSS}

The proposed band-stop FSS's unit cell is composed of two resonant elements, each one of them exhibits band-stop response over a part of the UWB band. The first resonant element is a square loop and the second element is a metallic ring. These elements shapes were chosen due to their ability to resonate at adjacent frequencies included in the UWB band. In order, to determine the design parameters that meet the objectives of the desired adjacent resonance frequencies and compact size unit cells, the composed elements were analyzed extensively. Fig. 2a shows the structure of the proposed UWB band-stop unit cell and its dimensions are $\mathrm{L} 1=8 \mathrm{~mm}$, $\mathrm{L} 2=6 \mathrm{~mm}, \mathrm{r} 1=3.1 \mathrm{~mm}, \mathrm{r} 2=2.5 \mathrm{~mm}$, and $\mathrm{g}=0.125 \mathrm{~mm}$. With these dimensions, the obtained S-parameters through the simulation are shown in Fig. 2b. From which, it can be seen that the transmission and reflection coefficients show a bandstop response over the entire UWB band. 


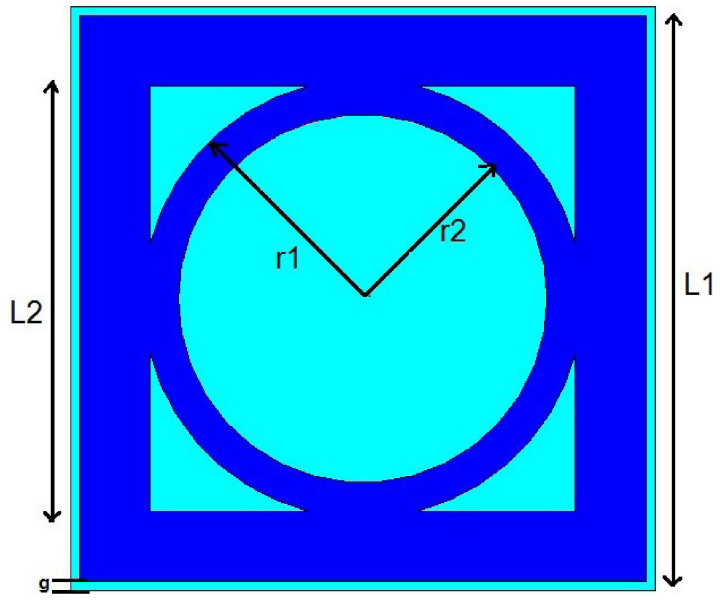

(a) Proposed band-stop unit cell

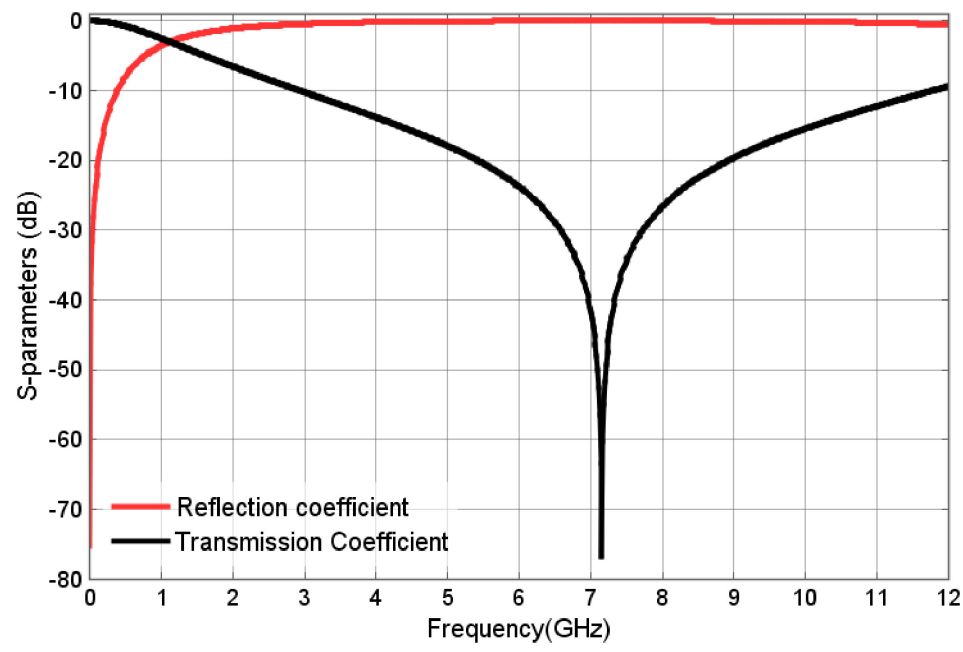

(b) S-parameters of the band-stop FSS

Fig. 2. Structure and results of the proposed band-stop FSS.

\section{Design of the proposed switchable FSS}

By replacing the ground plane, of the designed 3D band-pass FSS unit cell, with an interlayer composes of a number of the proposed band-stop FSS unit cells and integrating an on/off switch between the crossed tapered dipoles, as shown in Fig. 3a, a switchable band-stop/pass FSS can be obtained. This interlayer will act as a ground plane for the tightly coupled elements when the switch is off and as a reflector when the switch is turned on. As a result, it is possible to switch between UWB band-pass and UWB band-stop responses. Fig. $3 \mathrm{~b}$ illustrates the transmission coefficient of the proposed switchable FSS with the on and off states of the switch being modeled with and without a metallic pad, with dimensions $0.5 * 0.5 \mathrm{~mm}^{2}$, respectively. From which, we can notice that combining the two FSSs increases the lower and upper limits of the operating frequency band. This is because replacing the ground plane of the band-pass FSS with the UWB band-stop FSS changes its operating bandwidth. Also, the band-pass FSS layers and the transmission lines between them affect the operating band of the band-stop FSS. However, when the switch is on, the transmission coefficient is inferior to $-10 \mathrm{~dB}$ from $5 \mathrm{GHz}$ to 
$11 \mathrm{GHz}$, and it is $0 \mathrm{~dB}$, over the same band, when the switch is off. In other words, the proposed FSS acts as UWB band-stop, when the switch is on, and acts as UWB band-pass when the switch is off.

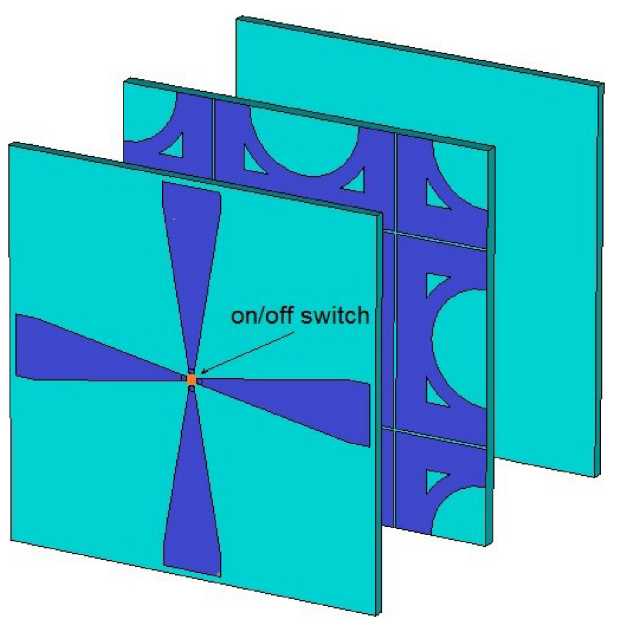

(a) Unit cell of the switchable FSS

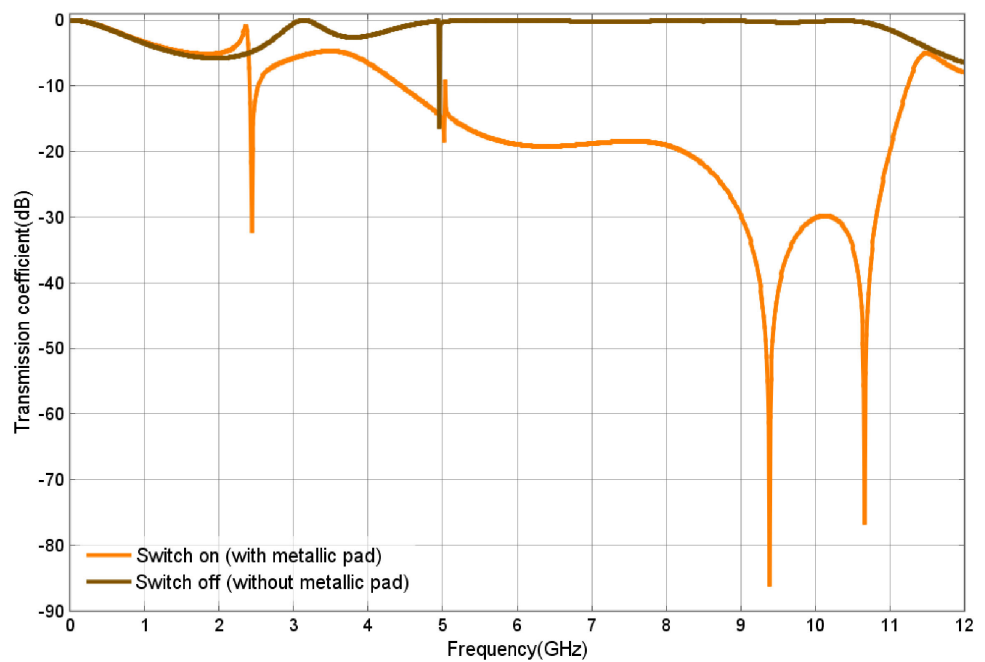

(b) Transmission coefficient of the Switchable FSS

Fig. 3. Structure and results of the proposed switchable FSS.

\section{Conclusion}

In this paper, planar band-stop, 3D band-pass and switchable UWB FSSs have been proposed. The main results that prove the design concept as well as the design process have also been clarified. The performance that can be provided by the proposed FSSs makes them good candidates for UWB applications such as radar and communications systems. Furthermore, by integrating the proposed switchable FSS with UWB antennas, antennas with reconfigurable radiation patterns and steering operation can be obtained. 